\title{
Realidad Virtual basada en un Vídeo de 360 grados y Una narración escrita para invitar a hombres a adoptar la Perspectiva de una Mujer Víctima de Acoso.
}

\section{Virtual Reality based on a 360-degree Video and A written narration to invite men to adopt The Perspective of a Female Victim of Harassment}

\author{
Sara Ventura ${ }^{1}$ \\ https://orcid.org/0000-0002-3851-7246 \\ Universidad de Bolonia, Italia \\ Georgina Cardenas ${ }^{2}$ \\ https://orcid.org/0000-0003-4569-1944 \\ Universidad Nacional Autónoma de México \\ Rosa Baños ${ }^{3}$ \\ https://orcid.org/0000-0003-0626-7665 \\ Universidad de Valencia, España
}

Recibido:11-05-2021

Aceptado: 29-08-2021

\section{Cita Recomendada}

Ventura, S., Cardenas, G. y Baños, R., (2021). Realidad Virtual basada en un vídeo de 360 grados, y una narración escrita para invitar a hombres a adoptar la perspectiva de una mujer víctima de acoso. Hamut' ay, 8 (2), 19-30, http://dx.doi.org/10.21503/hamu.v8i2.2284

\section{Resumen}

El presente artículo presenta el seguimiento de la perspectiva de una muestra masculina acerca de una mujer víctima de acoso de género en dos condiciones: un vídeo a 360-grados, y una narración escrita. El estudio tuvo como objetivo investigar sobre si las dimensiones psicológicas como la empatía, el machismo, la alexitimia y la actitud violenta, cambian después de tres meses de intervención. La muestra estuvo conformada por 35 hombres mexicanos que cumplieron ambas condiciones siguiendo un método contrabalanceado, quienes completaron cuestionarios para medir las dimensiones psicológicas en el momento previo a la intervención y posterior a ella. Los resultados mostraron cambios reflejados en el incremento en la empatía y una disminución en la actitud machista y violenta de los participantes, lo que llevó a concluir que el estudio abre nuevos caminos a posibles intervenciones para enfrentar el problema del acoso de género hacia las mujeres con la aplicación de las Tecnologías de la Información y la Comunicación (TIC).

Palabras Clave: acoso de género, cambio de actitud, víctima femenina, machismo, video 360-grados.

1. Doctora en psicología en la Universidad de Valencia (España), actualmente es investigadora y profesora en la Universidad de Bologna (Italia).sara91ventura@gmail.com.

2. Doctora en psicología, docente a tiempo completo de psicología en la Universidad Nacional Autónoma de México (México). cardenas.georgina@gmail.com

3. Doctora en Psicología, docente a tiempo completo de psicopatología en la Universidad de Valencia (España).rosa.banos@uv.es. 


\begin{abstract}
This article presents the follow-up of the perspective of a male sample about a female victim of gender harassment in two conditions: a 360-degree video, and a written narration. The study aimed to investigate whether psychological dimensions such as empathy, machismo, alexithymia and violent attitude change after three months of intervention. The sample consisted of 35 Mexican men who met both conditions following a counterbalanced method, who completed questionnaires to measure the psychological dimensions before and after the intervention. The results showed changes reflected in the increase in empathy and a decrease in the sexist and violent attitude of the participants, which led to the conclusion that the study opens new paths to possible interventions to face the problem of gender harassment towards women with the application of Information and Communication Technologies (ICT).
\end{abstract}

Key words: sexual harassment, attitudinal change, female victims, machismo, 360-degree video.

\section{Introducción}

El acoso de género es uno de los problemas mundiales que la sociedad debe enfrentar (Quick y McFadyen, 2017). En México, a finales de 2019 , se reportó que el $66.1 \%$ de las mujeres mayores de 15 años (casi 30.7 millones) han sido violadas por su esposo o su pareja. El acoso de género se produce cuando las personas son objeto de comentarios, gestos o acciones sexuales no deseadas, y las mujeres suelen ser las principales víctimas en espacios privados y/o públicos (Williams, 2018; Burn, 2019). Las mujeres víctimas perciben el acoso como algo molesto, humillante e intimidante, con el riesgo de comprometer de manera negativa su vida social y emocional (Gruber y Fineran, 2016). La literatura sobre el acoso de género subraya que la falta de empatía y, en concreto, la falta de toma de perspectiva está asociada al comportamiento de acoso (Barry et al., 2014). La empatía es un constructo multidimensional que consta de un componente cognitivo -la capacidad intelectual de comprender las emociones de otra persona sin dejar de ser un observador objetivo-, y un componente afectivo -el estado de verse afectado por las emociones de otra persona y compartirlas- (Harari et al., 2010; Baron-Cohen et al., 2013). Estudios preliminares han mostrado que los hombres que ofenden a las mujeres presentan menos empatía que los que tienen un comportamiento prosocial (Niedtfeld, 2017), y los individuos que tienen una baja capacidad de toma de perspectiva pueden malinterpretar las intenciones y los sentimientos de otras personas (Spataro et al., 2020). Debido a la enorme penetración del problema del acoso de género, y la relación con la empatía, se han desarrollado varios programas para prevenir y tratar tal comportamiento, basados principalmente en tomar la perspectiva de la mujer víctima a través de diversas actividades que incluyen videos, juegos de roles, la narración escrita, entre otros (Hunter, 2019).

El uso de las Tecnologías de la Información y la Comunicación (TIC), ha sido relevante para recrear situaciones problemáticas a través de estudios de caso o simulaciones que permiten enfrentar a los usuarios a temáticas específicas y llegar a hallazgos relevantes dentro del ámbito de la psicología; una de estas tecnologías es el vídeo a 360-grados. Es así, que en este estudio se trató de: 1) comprobar la hipótesis de si los cambios en las dimensiones de estado psicológico de empatía y actitud violenta cambian a lo largo de los tres tiempos (pre-post-test, y a los tres meses de seguimiento), y 2) comprobar la hipótesis si las dimensiones de rasgos psicológicos de empatía, machismo, y alexitimia cambian entre la preintervención y después de tres meses del estudio, a través de la aplicación del vídeo a 360-grados.

Dimensiones psicológicas: la empatía, el machismo, la alexitimia en la actitud violenta

El sentido de empatía juega un rol importante en los comportamientos prosociales y en la 
Realidad Virtual basada en un vídeo de 360 grados, y una narración escrita para invitar a hombres a adoptar la perspectiva de una mujer víctima de acoso.

prevención de aquellos actos violentos como el acoso de género. Además de la empatía, el trato machista de los hombres muestra ser una característica significativa en las actitudes violentas hacia las mujeres. El machismo es una exhibición de virilidad debida a la creencia de que el hombre es superior a la mujer (TerrazasCarrillo y Sabina, 2019).

La alexitimia, por otro lado, es un constructo psicológico que describe una condición de conciencia emocional reducida y que implica una incapacidad para reconocer y describir verbalmente los propios estados emocionales y los de los demás (Banzhaf et al., 2018). Según Vellotti et al., (2019) en su estudio refiere que las personas que puntuaban alto en alexitimia presentaban una baja capacidad empática, es decir, no reconocían el estado emocional de los demás.

Realidad Virtual basada en vídeo de 360-grados

La Realidad Virtual (RV) es una potente tecnología que permite crear y modificar mundos tridimensionales e inmersivos, y hasta ahora sus aplicaciones se han centrado fundamentalmente en simular la realidad externa, al hacer que la persona se sienta presente en un mundo que siente como "real", aunque no lo es (Botella et al., 2017). La adopción de la RV para estudiar y tratar trastornos psicológicos, ha obtenido resultados significativos (Ventura et al., 2018). El vídeo de 360 -grados se considera un subcampo de la RV en el que los contenidos pueden visualizarse en diferentes dispositivos como un ordenador portátil o un teléfono móvil, sumergiéndose totalmente en el escenario virtual con la ilusión de estar en ese entorno (Huang et al., 2017).

Recientemente, el vídeo a 360-grados se ha incluido como nuevo procedimiento para estudiar el acoso de género (Seinfeld et al., 2018; Steinfeld, 2020). Investigaciones muestran que adoptar la perspectiva de otra persona (es decir, imaginar cómo sería ser otra persona) puede ser un método eficaz para promover la empatía y motivar el comportamiento prosocial (Seinfeld et al., 2018; Bailenson et al., 2018; Ventura et al., 2020).
Las tareas de toma de perspectiva: el vídeo a 360 grados, y la narración escrita

En el presente estudio se adopta el enfoque del cambio de perspectiva a través de dos tareas: la RV basada en un vídeo de 360-grados, y una narración escrita para invitar a los hombres a adoptar la perspectiva de una mujer víctima de acoso.

El video de 360-grados fue grabado en diferentes lugares de la Ciudad de México donde ocurren varios escenarios de acoso, y refleja un día típico de una víctima de acoso de género. El primer escenario tuvo como objetivo generar la ilusión de encarnar el cuerpo de la víctima femenina siguiendo los movimientos corporales de la mujer que se presentan en el video (por ejemplo, mover sus manos hacia arriba y hacia abajo, acariciar sus extremidades y rotar sus manos). A continuación, el vídeo incluyó actividades de la vida cotidiana de la víctima (por ejemplo, ponerse los zapatos, desayunar, lavarse los dientes, preparar la mochila para ir a la universidad, ente otros), y varios escenarios de acoso que ocurren en diferentes lugares, como en la biblioteca de la universidad, en un taxi, en casa con la pareja, en el metro y durante el camino de vuelta a casa (Figura 1). La condición narrativa es la misma historia escrita en primera persona de manera que el lector se identifique con la protagonista de la historia.
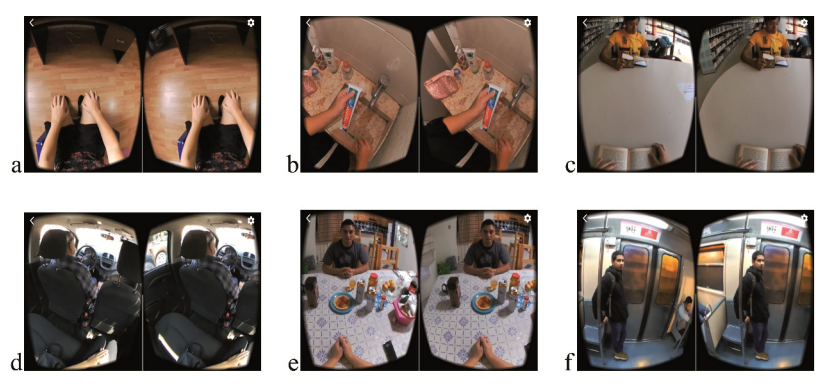

Nota. (a) = inducción a la personificación; (b) = actividades cotidianas; (c) = acoso en la biblioteca; (d) = acoso en el taxi; (e) = acoso en la pareja; (f) = acoso en el metro.

Figura 1. Capturas de pantalla de los escenarios de acoso de género en la experiencia de realidad virtual con vídeo a 360-grados. 
Materiales y métodos. Participantes

La muestra estuvo compuesta por 35 participantes. Los criterios de exclusión fueron: (1) ser menor de $\leq 18$ años, (2) tener problemas físicos que pudieran inhibir los movimientos libres, (3) antecedentes de acoso con consecuencias legales, (4) uso o abuso de drogas y, (5) estar bajo tratamientos psicológicos. Las estadísticas descriptivas de las medidas sociodemográficas se describen en la Tabla 1.

Tabla 1. Estadísticas descriptivas de las medidas sociodemográficas $(\mathrm{N}=35)$

\begin{tabular}{lcc}
\hline & $\boldsymbol{M}(\boldsymbol{S D})$ & $\%$ \\
\hline Edad & $26.31(7.56)$ & - \\
Educación & & \\
Secundaria & - & $8.6 \%$ \\
Grado & - & $71.4 \%$ \\
Máster & - & $20.0 \%$ \\
Antecedentes de enfermedades mentales o crónicas (\% si) & - & $11.1 \%$ \\
Consumo de alcohol & & \\
$\quad$ Nunca & - & $28.6 \%$ \\
$\quad$ Una vez al mes & - & $31.4 \%$ \\
$\quad$ 2-4 veces al mes & - & $34.3 \%$ \\
$\quad$ 2-3 veces a la semana & - & $5 . \%$ \\
Problema legal por acoso sexual (\% si) & - & $0.0 \%$ \\
\hline
\end{tabular}

Fuente: Elaboración propia (2021)

\section{Instrumentos}

Para la recolección de datos cuantitativos se utilizaron seis instrumentos. El primero fue la escala de Machismo y Caballerismo (MCS) (Arciniega y otros, 2008). Escala de autoinforme mexicana validada con 20 ítems $(1=$ muy en desacuerdo; $7=$ muy de acuerdo $)$ que mide los constructos de "machismo" (poder y actitudes agresivas de los hombres) y caballerismo (conexión emocional, honor y crianza de los hombres). La consistencia interna es adecuada para la recolección de datos, siendo la confiabilidad machismo $(\alpha=0.75)$ y caballerismo $(\alpha=0.83)$. El segundo fue el Índice de Reactividad Interpersonal (IRI) (Davis 1980; Pérez-Albéniz et al., 2003). Escala de autoinforme con 28 ítems ( $1=$ muy en desacuerdo; $7=$ muy de acuerdo), adaptados de la escala original (Davis, 1980). Se compone de cuatro factores: toma de perspectiva, fantasía, preocupación empática y malestar personal. La consistencia interna es adecuada para todas las subescalas de este estudio: toma de perspectiva ( $\alpha=0.78)$, fantasía ( $\alpha=0.89$ ), preocupación empática $(\alpha=$ $0.72)$ y angustia personal $(\alpha=0.89)$. El tercero fue la Escala de Alexitimia de Toronto (TAS-20) (Taylor et al., 1985; Moral de la Rubia, 2008). Es una escala mexicana con 20 ítems ( $1=$ muy en desacuerdo; 7 = muy de acuerdo) que mide tres factores: dificultad para expresar sentimientos, dificultad para identificar sentimientos $y$ pensamiento orientado. La consistencia interna es adecuada para las tres subescalas, las cuales tienen una confiabilidad de: dificultad para explicar sentimientos $(\alpha=0.89)$, dificultad para identificar sentimientos $(\alpha=0.94)$ y pensamiento extremadamente orientado hacia el exterior ( $\alpha$ $=0.70)$. El cuarto instrumento fue la Escala de Actitud hacia la Violencia de Género (ATG-S). Se trata de un autoinforme ad-hoc con 5 ítems ( $1=$ nada; 5 = totalmente), basado en la escala original de Sexismo Ambivalente (Glick y Fiske, 1996). El quinto instrumento fue la Escala de empatía (ES). Se trata de un autoinforme ad hoc que contiene 5 ítems ( $1=$ nada; $5=$ totalmente) que miden el estado de empatía (Seinfeld et al., 2018). Y el sexto instrumento fue la Escala del sentido de la corporeidad y del sentido de la presencia (SOE-SOP): Se trata de un autoinforme con 13 ítems valorados en una escala de 7 puntos ( 1 = totalmente en desacuerdo; $7=$ totalmente de acuerdo). Diez ítems fueron adaptados del cuestionario original de Longo para evaluar la propiedad, la localización y la agencia del cuerpo femenino (Longo, 2008) ( $\alpha=0.88$ ); tres ítems fueron desarrollados por los autores para evaluar el sentido de presencia que se siente durante toda la experiencia de inmersión (Usoh et al., 2000), con este último se recopilaron datos sobre la tecnología.

Para recopilar la información cualitativa se utilizó un cuestionario de preguntas abiertas para explorar el impacto de la intervención durante los tres meses, y el tipo de sentimientos que experimentaron los participantes: $(\mathrm{P} 1)_{\dot{ }} \mathrm{A}$ lo largo de estos meses, has pensado en el experimento de acoso de género en el que participaste?, (P2) 
¿Has tenido algún pensamiento o sentimiento negativo después de tu participación en el estudio?, (P3) ¿Has tenido algún pensamiento o sentimiento positivo después de tu participación en el estudio?, (P4) ¿Has tenido pensamientos o sentimientos extraños, inusuales o inesperados en relación al estudio de acoso de género en el que participaste?, (P5) ¿Sientes que tu actitud hacia la mujer ha cambiado después de tu participación en el estudio?.

\section{Procedimiento}

El experimento se desarrolló en la Universidad Nacional Autónoma de México (Ciudad de México). Los participantes primero firmaron el consentimiento informado, y respondieron los cuestionarios de línea base (T1) (MCS, IRI, TAS-20, ATG-S, ES-S), luego fueron contrabalanceados en las dos condiciones para evitar el efecto primario/recencia: el grupo A siguieron el orden video a 360-grados - narrativa, y el grupo B siguieron el orden de la narrativa - video a 360-grados. En ambas condiciones, los participantes adoptaron la perspectiva de la mujer para experimentar lo que es ser una mujer víctima de acoso sexual en primera persona. En la condición de vídeo a 360-grados, los participantes realizaron primero una tarea de encarnación para darles la ilusión de tener un cuerpo femenino, y luego fueron inmersos en varios escenarios de acoso por casi 20 minutos. En la condición narrativa, los participantes leyeron la historia con la instrucción de imaginar el contenido como si les estuviera ocurriendo a ellos, esta tarea duró 10 minutos. Después de ambas condiciones, los participantes respondieron a las escalas ATG-S y ES-S (T2) para medir el estado psicológico de la actitud violenta y de la empatía.

Tras tres meses del estudio (T3), los participantes volvieron a rellenar el MCS, el IRI, el TAS-20, que miden los rasgos psicológicos, junto a los cuestionarios ATG-S y ES-S, y las preguntas abiertas.

Después de ejecutado el experimento se realizó los análisis estadísticos con el SPSS v.26. Se realizó un ANOVA con el factor tiempo (T1, T2 y T3) como análisis intra-sujetos para analizar el efecto de la intervención sobre la empatía (ES-S) y la actitud violenta (ATG-S). Además, se realizaron pruebas $t$ para evaluar la diferencia entre el pretest y a los tres meses de seguimiento para las escalas TAS-20, IRI y MCS. Se realizaron también pruebas t para analizar el aspecto tecnológico del entorno, es decir, la sensación de corporeidad y la presencia. Las preguntas abiertas se abordaron mediante el análisis de contenido para identificar las principales categorías conceptuales y las respectivas subcategorías. Por último, se abstrajo el significado general (White y Marsh, 2006).

\section{Confidencialidad o Consentimiento informado}

El presente estudio ha sido aprobado por el Comité de Ética de la Universidad Nacional Autónoma de México (UNAM) con la clave EP/ PMDPSIC/0151/19 (Anexo 1).

\section{Resultados}

El análisis cuantitativo se realizó un primer análisis sobre las estadísticas descriptivas del IRI, el TAS-20, el MCS, el ATG-S y el ES-S como se muestran en la Tabla 2.

Tabla 2. Estadísticas descriptivas de las medidas relacionadas $(\mathrm{N}=35)$.

\begin{tabular}{lcc}
\hline & $\begin{array}{c}\text { Línea base } \\
(\mathbf{n}=\mathbf{3 5}) \\
\boldsymbol{M}(\boldsymbol{S D})\end{array}$ & $\begin{array}{c}\text { Seguimiento } \\
(\mathbf{n}=\mathbf{3 5}) \\
\boldsymbol{M}(\boldsymbol{S D})\end{array}$ \\
\hline $\begin{array}{l}\text { Machismo y Caballerismo (MCS) } \\
\text { Machismo }\end{array}$ & $1.96(0.77)$ & $1.75(0.60)$ \\
Caballerismo & $5.36(0.91)$ & $5.36(0.98)$ \\
Empatía (IRI) & & \\
Toma de perspectiva & $4.78(0.80)$ & $4.92(0.73)$ \\
Fantasía & $4.58(1.02)$ & $4.84(1.21)$ \\
Empatía cognitiva & $5.24(0.83)$ & $5.31(0.73)$ \\
Distrés empático & $3.21(1.19)$ & $3.09(1.26)$ \\
Alexitimia (TAS-20) & & \\
Dificultad para expresar sentimientos & $3.74(1.54)$ & $3.68(1.45)$ \\
Pensamiento orientado al exterior & $2.49(0.73)$ & $2.93(0.66)$ \\
Dificultad para identificar los sentimientos & $2.95(1.55)$ & $2.72(1.39)$ \\
Actitud Violenta (ATG-S) & $2.26(0.57)$ & $1.25(0.38)$ \\
\hline
\end{tabular}

Nota. MCS = Escala de Machismo y Caballerismo; IRI = Índice de Reactividad Interpersonal; TAS-20 = Escala de Alexitimia de Toronto; ATG-S = Escala de Actitud hacia la Violencia de Género. Fuente: Elaboración propia (2021) 
Los resultados del análisis ANOVA mostraron una diferencia significativa en la puntuación ATG-S de T1 a T2: p < 0.001, IC del 95\% (0.844, 1.224), y de T1 a T3: p < 0.001, IC del 95\% $(0.758,1.265)$. Además, los resultados mostraron una diferencia significativa también para la puntuación de ES-S de T1 a T2: p < 0.001, IC del $95 \%(-0.769,-0.293)$, y de T1 a T3: $\mathrm{p}=0.047$, IC del 95\% (-0.421, -0.002) (Figura 2).
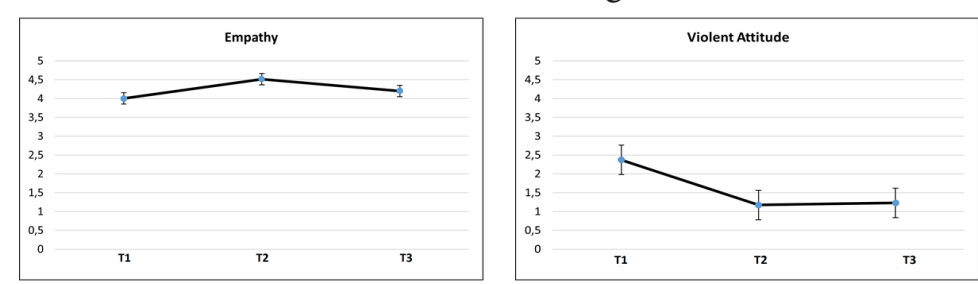

Figura 2. Gráficos de barras simples para la empatía y la actitud violenta a lo largo de tres tiempos (T1, T2 y T3).

Nota: Empatía: T1: $M=4, T 2: M=4,51, T 3: M=4,2$; Actitud violenta: T1: $\mathrm{M}=2,26, \mathrm{~T} 2: \mathrm{M}=1,23, \mathrm{~T} 3: \mathrm{M}=1.25$.

Los resultados indicaron que no hay diferencias significativas para la escala IRI: toma de perspectiva $\mathrm{t}(34)=-1.352, \mathrm{p}=0.185, \mathrm{~d}=0.21$, IC 95\% $(-0.368,0.074)$, fantasía $\mathrm{t}(34)=-1.777$ $, \mathrm{p}=0.085, \mathrm{~d}=1.20$, IC 95\% (-0.551, 0.037), preocupación empática $\mathrm{t}(34)=-0.641, \mathrm{p}=0.526$, $\mathrm{d}=0.19$, IC 95\% $(-0.298,0.155)$, angustia personal $\mathrm{t}(34)=0.898, \mathrm{p}=0.376, \mathrm{~d}=0.10$, IC 95\% $(-0.156$, 0.404); ninguna diferencia significativa para el machismo $\mathrm{t}(34)=1.812, \mathrm{p}=0.079, \mathrm{~d}=0.27, \mathrm{IC}$ $95 \%(-0.026,0.449)$. Además, los resultados no indicaron diferencias significativas en la escala de alexitimia para la subescala de dificultad que explica los sentimientos $\mathrm{t}(34)=0.281, \mathrm{p}=0.780$, $\mathrm{d}=0.18$, IC 95\% (-0.391, 0.517), y la dificultad para identificar los sentimientos $\mathrm{t}(34)=1.831$, $\mathrm{p}=0.076, \mathrm{~d}=0.22,95 \% \mathrm{CI}(-0.025,0.482)$; se encontró una diferencia significativa para la subescala de pensamiento extremadamente orientado $\mathrm{t}(34)=-4.996, \mathrm{p}<0.001, \mathrm{~d}=0.11,95 \%$ CI $(-0.618,-0.261)$. Por último, los resultados indicaron una diferencia significativa para la medida de actitud violenta $\mathrm{t}(34)=10.041, \mathrm{p}<$ $0.001, \mathrm{~d}=1.71,95 \%$ CI $(0.807,1.216)$.

Los resultados sobre la tecnología del video a 360-grados indicaron una puntuaciones de encarnación significativamente mayores que el nivel de azar de 4 para la localización $(M=5.82$,
$\mathrm{SD}=1.33), \mathrm{t}(43)=9.04, \mathrm{p}<0.001, \mathrm{y}$ la propiedad $(\mathrm{M}=5.12, \mathrm{SD}=1.10), \mathrm{t}(43)=6.79, \mathrm{p}<0.001$; pero no para la agencia $(\mathrm{M}=4.28, \mathrm{SD}=1.50), \mathrm{t}(43)=$ $1.24, \mathrm{p}=0,222$. Las puntuaciones en el sentido de presencia $(\mathrm{M}=5.23, \mathrm{SD}=1.00)$ también fueron mayores que el nivel de azar de $4, \mathrm{t}(43)=8.23$, $\mathrm{p}$ $<0.001$.

Del análisis de contenido de las preguntas abiertas surgieron las siguientes temáticas (Tabla 3).

Tabla 3. Porcentaje de emociones experimentadas por los participantes y las respectivas subcategorías $(\mathrm{N}=35)$.

\begin{tabular}{lc}
\hline Categorías & $\boldsymbol{n}(\%)$ \\
Subcategorías & $\%$ \\
\hline Pensamientos positivos & $31(100 \%)$ \\
Empatía & $52.32 \%$ \\
Compasión & $30.25 \%$ \\
Concienciación & $16.43 \%$ \\
Pensamientos negativos & $21(100 \%)$ \\
Incómodo (falta de confort) & $23.80 \%$ \\
Tristeza & $14.33 \%$ \\
Rabia & $9.5 \%$ \\
Soledad & $14.28 \%$ \\
Impotencia & $38.09 \%$ \\
Cambio de actitud & $27(100 \%)$ \\
Activismo & $76.42 \%$ \\
Violencia & $23.58 \%$ \\
\hline
\end{tabular}

Fuente: Elaboración propia (2021).

\section{Resultados cualitativos}

Con relación a los resultados cualitativos, los 31 participantes dijeron sentirse más empáticos con las mujeres, pensamientos positivos. Gracias a las actividades de toma de perspectiva realizadas durante el estudio, los participantes informaron de que se ponían en la perspectiva de las mujeres, y ahora saben cómo podrían sentirse las mujeres en determinadas circunstancias:

"Me siento empático y sensible para prevenir y actuar en situaciones de acoso, percibo la necesidad y el deseo de transformar la realidad social mexicana" (participante 1).

"Ahora, trato de ser más empático con las mujeres" (participante 2).

"Me siento más empático con las protestas de las mujeres, y creo que puedo, al menos, hacer un cambio en mi entorno" (participante 3 ). 
Realidad Virtual basada en un vídeo de 360 grados, y una narración escrita para invitar a hombres a adoptar la perspectiva de una mujer víctima de acoso.

El sentimiento de compasión también surgió desde los análisis. Los participantes declararon que, gracias al estudio, pudieron reconocer las circunstancias en las que las mujeres se sienten incómodas, y ahora intentan aliviar el sufrimiento femenino evitando esos comportamientos:

\footnotetext{
"Gracias al estudio, he podido captar algunas circunstancias en las que las mujeres pueden sentirse incómodas, y esto me ayuda a saber cuándo puedo ayudarlas" (participante 4).

"Ahora trato de entender la violencia que sufre la mujer, y trato de ayudarlas, y explicarles que no están solas y que pueden tener mi apoyo" (participante 5).
}

Por último, el estudio ayudó a los participantes a ser más conscientes del problema del acoso. Tras adoptar la perspectiva de la víctima, son más conscientes de lo que significa ser víctima del acoso:

"El experimento me dio la posibilidad de ver otra perspectiva de la situación de acoso, es muy fácil escuchar noticias sobre el acoso, pero el estudio ayuda mucho a poder ver la situación desde la perspectiva de una mujer. Como hombre, es difícil vivir estas situaciones a menos que el estudio me lo muestre" (participante 6).

"Antes de participar en el estudio, para mí era habitual mirar a las mujeres, creía que no era violento, que incluso era normal, y ahora soy un poco más consciente de que es como conceptualizar a la mujer como un objeto" (participante 7).

Con relación a los Pensamientos negativos. Las respuestas se centraron sobre todo en la sensación de impotencia de los participantes para hacer frente a los problemas de acoso. Veintiún participantes dijeron que querían hacer algo para mejorar la situación social de las mujeres, pero se sentían impotentes y solos luchando contra el problema:

"Siento que no puedo hacer nada" (participante 8).

"Tengo sentimientos de aislamiento y de lucha por una sociedad mezquina" (participante 9).

Además, después de adoptar la perspectiva de la víctima, los participantes se sintieron tristes y enfadados porque lo que percibieron durante la actividad de adopción de la perspectiva, podría ocurrir en su familia, a su hermana, sobrina o cónyuge.

Cambio de actitud. Después de la tarea, 27 participantes dijeron que revalorizaron su actitud violenta hacia las mujeres, es decir, que fueron más amables, en particular no expresaron comentarios sexuales hacia las mujeres cuando estaban con amigos porque ahora entienden cómo se pueden sentir las mujeres:

"Presto más atención a mi comportamiento con las mujeres, incluso en el trabajo comparto lecturas con otros hombres para entender la perspectiva femenina" (participante 10).

"El experimento me ayudó a pensar más en mi punto de vista y a analizar más las situaciones" (participante 11).

Otro resultado importante surgido del análisis de contenido es la subcategoría del activismo social. Después de la tarea, las participantes dijeron que ahora estaban más involucradas con los movimientos feministas, que participaban en las manifestaciones sociales como el 8 de marzo, $y$ que daban más valor a estas iniciativas:

"Entiendo más el objetivo de los movimientos feministas desde la perspectiva de las chicas que lo llevan a cabo" (participante 12).

"Ahora, cuando veo una manifestación femenina, estoy a favor y entiendo por qué lo hacen" (participante 13).

\section{Discusión y Conclusiones}

Después del estudio realizado se contrastó que el cambio de perspectiva de los hombres hacia una mujer víctima de acoso fue inducido a través del sistema de video a 360-grados, y de la imaginación mediante la lectura de una narración escrita. Se obtuvieron resultados relevantes de las variables de empatía y actitud violenta, que cambian significativamente a lo largo de los tres tiempos del estudio. En particular, la variable de estado de empatía aumenta significativamente desde antes hasta después de la intervención, y la variable siguió siendo alta después de tres meses en comparación con la línea base. La misma tendencia se encontró para la variable de actitud violenta, es decir, disminuyó significativamente 
desde antes hasta después de la intervención, y la variable siguió siendo significativamente menor después de los tres meses. Estos resultados son interesantes porque muestran el potencial de la intervención de toma de perspectiva para cambiar el sentimiento y la conciencia hacia el problema del acoso de género contra las mujeres, en consistencia con estudios anteriores que presentan cómo la toma de perspectiva podría cambiar la actitud hacia un grupo externo (Herrera et al., 2018). Respecto a los resultados a lo largo de los dos tiempos del estudio (línea-base y seguimiento), no hay diferencias significativas para la empatía, evaluada con el IRI (PérezAlbéniz, 2003). Sin embargo, se encontró una tendencia (marginalmente significativa) en la disminución del rasgo de machismo después de tres meses. Este es un resultado interesante porque subraya el potencial de la intervención para cambiar los rasgos masculinos (Steinfeld, 2020). Además, los resultados del machismo podrían ser también moderados por la situación social e histórica que vive México con el problema del feminicidio. Desde hace tiempo, y especialmente durante los tres meses posteriores al estudio (enero-marzo de 2020), la Ciudad de México sufrió un movimiento feminista (Sepúlveda, 2018). El movimiento social, y la cantidad de noticias sobre el tema, han podido influir en la dimensión del machismo, y en cómo los hombres perciben el acoso de género. Los estudios futuros deberían tener en cuenta el compromiso de los participantes con las campañas feministas. Además, los resultados mostraron diferencias significativas para la subescala "pensamiento extremadamente orientado" de la escala de alexitimia, esto significa que los participantes, después del estudio, son más capaces de estar centrados en sus pensamientos y sentimientos. Este resultado coincide con los obtenidos en las preguntas abiertas. De hecho, del análisis cualitativo de los contenidos, se desprende que los participantes son más conscientes de sus sentimientos hacia la mujer en general, y hacia su familia (cónyuge, hermana, sobrina) en particular. Después de tres meses, los participantes afirmaron que se sienten más cercanos a las mujeres, que comprenden cómo pueden sentirse las mujeres en determinadas circunstancias $y$ que intentan evitar situaciones que puedan inducir a una mujer a sentirse incómoda. $\mathrm{La}$ atención, y la empatía hacia las mujeres podría haberles ayudado a estar más centrados en sus sentimientos. Este resultado podría abrir la dirección futura del entrenamiento de la empatía y la compasión para mejorar la autoconciencia de los hombres hacia los sentimientos de las mujeres (Gilbert, 2018).

El estudio presentó también algunas limitaciones, en primer lugar, siguió un método contrabalanceado, es decir, a los participantes se les administraron ambas condiciones (vídeo a 360-grados y narración), y por eso no fue posible conocer el peso específico de las dos condiciones en los cambios observados. Los estudios futuros deberían adoptar un método de ensayo de control aleatorio para evaluar qué condición entre el vídeo a 360-grados y la narración es mejor para provocar un cambio positivo en la empatía y las variables relacionadas. En segundo lugar, el tamaño del efecto fue pequeño para la mayoría de las medidas. En tercer lugar, la muestra actual estaba compuesta mayoritariamente por estudiantes universitarios, y la investigación futura podría dirigirse a poblaciones como hombres en prisión, hombres mayores, entre otras. Por último, la consistencia interna del autoinforme de actitud violenta es limitada.

En conclusión, el acoso de género es un problema social importante en muchos países. En México, datos del Secretariado Ejecutivo del Sistema Nacional de Seguridad Pública muestran que en 2016 hubo aproximadamente 29.725 carpetas de investigación por delitos de feminicidio (Melgar, 2019). Debido a esta gran penetración del problema, es necesario proponer y probar soluciones eficaces para esta problemática. A pesar de las limitaciones, el estudio abrió nuevas direcciones para el mecanismo de toma de perspectiva, para desarrollar programas educativos basados en la prevención, y para generar intervenciones que permita a los acosadores enfrentarse al problema del acoso de género. 
Realidad Virtual basada en un vídeo de 360 grados, y una narración escrita para invitar a hombres a adoptar la perspectiva de una mujer víctima de acoso.

\section{Agradecimiento}

Este trabajo ha sido apoyado por la subvención BEFPI-2019 - Generalitat Valenciana (España), CIBEROBN, una iniciativa del ISCIII (ISC III CB06 03/0052) y PROMETEO ( P R O METEO/2018/110/Conselleria de Educación, Investigación, Cultura I Deporte, Generalitat Valenciana).

\section{Referencias Bibliográficas}

Arciniega, G. M., Anderson, T. C., Tovar-Blank, Z. G., \& Tracey, T. J. (2008). Toward a fuller conception of Machismo: Development of a traditional Machismo and Caballerismo Scale. Journal of Counseling Psychology, 55(1), 19-33. https:// doi.org/10.1037/0022-0167.55.1.19

Bailenson, J. (2018). Experience on demand: What virtual reality is, how it works, and what it can do. Norton \& Company.

Banzhaf, C., Hoffmann, F., Kanske, P., Fan, Y., Walter, H., Spengler, S., \& Bermpohl, F. (2018). Interacting and dissociable effects of alexithymia and depression on empathy. Psychiatry research, 270, 631-638.

https://doi.org/10.1016/j.psychres.2018.10.045

Baron-Cohen, S., Tager-Flusberg, H., \& Lombardo, M. (2013). Understanding other minds: Perspectives from developmental social neuroscience. Oxford university press.

https: / / doi.org/10.1093/acprof:oso/9780199692972.001.0001

Barry, C. T., Kauten, R. L., \& Lui, J. H. (2014). Self-perceptions of empathy and social support as potential moderators in the relation between adolescent narcissism and aggression. Individual Differences Research, 12(4-A), 170-179.

Botella, C., Fernández-Álvarez, J., Guillén, V., García-Palacios, A., \& Baños, R. (2017). Recent progress in virtual reality exposure therapy for phobias: a systematic review. Current psychiatry reports, 19(7), 1-13.

https://doi.org/10.1007/s11920-017-0788-4

Burn, S. M. (2019). The psychology of sexual harassment. Teaching of Psychology, 46(1), 96-103. https://doi.org/10.1177/0098628318816183
Cameron, C. D., Hutcherson, C. A., Ferguson, A. M., Scheffer, J. A., Hadjiandreou, E., \& Inzlicht, M. (2019). Empathy is hard work: People choose to avoid empathy because of its cognitive costs. Journal of Experimental Psychology: General. https://doi.org/10.1037/xge0000595

Davis, M. H. (1980). A multidimensional approach to individual differences in empathy. Day, A., Casey, S., \& Gerace, A. (2010). Interventions to improve empathy awareness in sexual and violent offenders: Conceptual, empirical, and clinical issues. Aggression and Violent Behavior, 15(3), 201-208.https://doi.org/10.1016/j. avb.2009.12.003

De Borst, A. W., Sanchez-Vives, M. V., Slater, M., \& de Gelder, B. (2020). First person virtual embodiment modulates cortical network that encodes the bodily self and its surrounding space during the experience of domestic violence. eNeuro, 7(3), 1-14.

https://doi.org/10.1523/ENEURO.0263-19.2019

De la Peña, N., Weil, P., Llobera, J., Giannopoulos, E., Pomés, A., Spanlang, B., Friedman, D., Sanchez-Vives, M., \& Slater, M. (2010). Immersive journalism: immersive virtual reality for the first-person experience of news. Presence: Teleoperators and virtual environments, 19(4), 291-301. https://doi.org/10.1162/PRES_a_00005 Gilbert, P. (2018). Practice quality and effectiveness of a compassion training intervention: The Importance of embodying the compassionate self.

Glick P, Fiske S. T. (1996). The ambivalent sexism inventory: Differentiating hostile and benevolent sexism. Journal of Personality and Social Psychology, 10(3), 491-512).

https://doi.org/10.1037/0022-3514.70.3.491

Gobierno de México (s.f.). https://www.gob.mx/ sesnsp

Gruber, J., \& Fineran, S. (2016). Sexual harassment, bullying, and school outcomes for high school girls and boys. Violence Against Women, 22(1), 112-133.

https://doi.org/10.1177/1077801215599079

Harari, H., Shamay-Tsoory, S. G., Ravid, M., \& Levkovitz, Y. (2010). Double dissociation between cognitive and affective empathy in bor- 
derline personality disorder. Psychiatry research, 175(3), 277-279. https://doi.org/10.1016/j.psychres.2009.03.002

Herrera, F., Bailenson, J., Weisz, E., Ogle, E., \& Zaki, J. (2018). Building long-term empathy: A large-scale comparison of traditional and virtual reality perspective-taking. PloS one, 13(10). https://doi.org/10.1371/journal.pone.0204494

Huang, J., Chen, Z., Ceylan, D., Jin, H. (2017). 6-DOF VR videos with a single 360-camera. In IEEE Virtual Reality (VR) (pp. 37-44).

https://doi.org/10.1109/VR.2017.7892229

Hunter, K. B . (2019). Relapse prevention and safety planning: A viable short-term intervention. Practice, 7(1).

Longo, M. R., Schüür, F., Kammers, M. P., Tsakiris, M., Haggard, P. (2008). What is embodiment? A psychometric approach. Cognition, 107(3), 978-998.

https://doi.org/10.1016/j.cognition.2007.12.004 Melgar, L. (2019). Reclaiming the Streets: Feminicidio and the Space of Women's Rights in Mexico. In Negotiating Space in Latin America (pp. 43-65). Brill Rodopi..

https://doi.org/10.1163/9789004408708_004

Moral de la Rubia, J. (2008). Propiedades psicométricas de la Escala de Alexitimia de Toronto de 20 reactivos en México. Revista Electrónica de Psicología Iztacala, 11(2), 97-114.

Niedtfeld, I. (2017). Experimental investigation of cognitive and affective empathy in borderline personality disorder: effects of ambiguity in multimodal social information processing. Psychiatry research, 253, 58-63.

https://doi.org/10.1016/j.psychres.2017.03.037

Pérez-Albéniz, A., De Paúl, J., Etxeberría, J., Montes, M. P., \& Torres, E. (2003). Adaptación de interpersonal reactivity index (IRI) al español. Psicothema, 15(2), 267-272.

Quick, J. C., \& McFadyen, M. (2017). Sexual harassment: Have we made any progress? Journal of occupational health psychology, 22(3), 286-298. https://doi.org/10.1037/ocp0000054

Sánchez de los Monteros Arriaga, A. C. (2020). La violencia de género en México ¿en qué vamos? Revista Digital Universitaria, 21(4).

h t t p s : / / d o i.org/ $10.22201 /$ cuaieed.16076079e.2020.21.4.1

Seinfeld, S., Arroyo-Palacios, J., Iruretagoyena, G. Hortensius, L. E., Zapata, D. Borland, B. de Gelder, Slater, M., \& Sanchez-Vives, MV., (2018). Offenders become the victim in virtual reality: impact of changing perspective in domestic violence. Scientific reports, 8(1), 1-11. https://doi. org/10.1038/s41598-018-19987-7

Sepúlveda, G. A. (2018). Activist Archiving and the Feminist Movement in Mexico: Collecting Art and Ephemera as Political Practice. Unsettling Activisms: Critical Interventions on Aging, Gender, and Social Change, 194.

Spataro, P., Calabrò, M., \& Longobardi, E. (2020). Prosocial behaviour mediates the relation between empathy and aggression in primary school children. European Journal of Developmental Psychology, 17(5), 727-745.

https://doi.org/10.1080/17405629.2020.1731467

Steinfeld, N. (2020). To Be there when it Happened: Immersive Journalism, Empathy, and Opinion on Sexual Harassment. Journalism Practice, 14(2), 240-258.

https://doi.org/10.1080/17512786.2019.1704842 Taylor, G. J., Ryan, D., \& Bagby, M. (1985). Toward the development of a new self-report alexithymia scale. Psychotherapy and psychosomatics, 44(4), 191-199.

https://doi.org/10.1159/000287912

Terrazas-Carrillo, E., \& Sabina, C. (2019). Dating violence attitudes among Latino college students: An examination of gender, machismo, and marianismo. Violence and victims, 34(1), 194-210. https://doi.org/10.1891/0886-6708.VV-D-1700172

Usoh, M., Catena, E., Arman, S., \& Slater, M. (2000). Using presence questionnaires in reality. Presence: Teleoperators \& Virtual Environments, 9(5), 497-503.

https://doi.org/10.1162/105474600566989

Velotti, P., Garofalo, C., Dimaggio, G., \& Fonagy, P. (2019). Mindfulness, alexithymia, and empathy moderate relations between trait aggression and antisocial personality disorder traits. Mindfulness, 10(6), 1082-1090. https://doi.org/10.1007/s12671-018-1048-3 Ventura, S., Badenes-Ribera, L., Herrero, R., 
Realidad Virtual basada en un vídeo de 360 grados, y una narración escrita para invitar a hombres a adoptar la perspectiva de una mujer víctima de acoso.

Cebolla, A., Galiana, L., \& Baños, R. (2020). Virtual reality as a medium to elicit empathy: a meta-analysis. Cyberpsychology, Behavior, and Social Networking, 23(10), 667-676. https://doi. org/10.1089/cyber.2019.0681

Ventura, S., Baños, R. M., Botella, C., \& Mohamudally, N. (2018). Virtual and augmented reality: New frontiers for clinical psychology. In State of the art virtual reality and augmented reality knowhow (pp. 99-118).

https://doi.org/10.5772/intechopen.74344

White, M. D., \& Marsh, E. E. (2006). Content analysis: A flexible methodology. Library trends, 55(1), 22-45.

https://doi.org/10.1353/lib.2006.0053

Williams, C. L. (2018). Sexual harassment in organizations: A critique of current research and policy. In Sexual Harassment and Sexual Consent (pp. 20-43). Routledge.

https://doi.org/10.4324/9781315129259-3 


\title{
Anexo 1 Aprobación comité de ética.
}

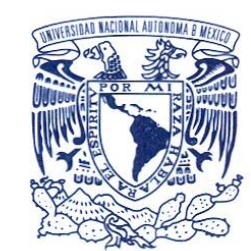

VNIVER'IDAD NACIONAL

AVTONOMA DE

MEXICOO

\section{Estudios de Posgrado Programa de Maestría y Doctorado en Psicología Coordinación}

\author{
Folio: EP/PMDPSIC/0151/19
}

SARA VENTURA

ALUMNO (A) DE DOCTORADO

PROGRAMA DE MAESTRÍA Y DOCTORADO EN PSICOLOGÍA

$P R E S E N T E$

Por medio de la presente hago llegar a usted el dictamen de la Comisión de Ética, asignada por el Comité Académico de este Programa, sobre el protocolo de investigación "Evaluación de un procedimiento basado en tecnología inmersiva con cámara $360^{\circ}$ para estudiar el sentido de empatía en comparación con un procedimiento tradicional en población mexicana. Un estudio exploratorio para enfrentar el problema del acoso de género".

Consideraciones:

Las normas éticas de la APA (Ethical Principles of Psychologists and Code of Conduct // http://www.apa.org/ethics/code/index.aspx ), señalan 5 principios generales básicos, a saber: 1. Beneficiar y no perjudicar, 2. Fidelidad y responsabilidad, 3. Integridad, 4. Justicia y 5. Respeto a las personas y la dignidad. No se oberva que el proyecto de investigación sometido a dictamen viole alguno de ellos.

En virtud de lo anterior la comisión no aprecia que haya elementos de objeción ética para impugnar el protocolo de investigación utilizado, por lo que emite un DICTAMEN FAVORABLE para su realización.

Sin otro particular por el momento, envío a usted un cordial saludo, reiterándole mi más alta consideración académica y personal estima.

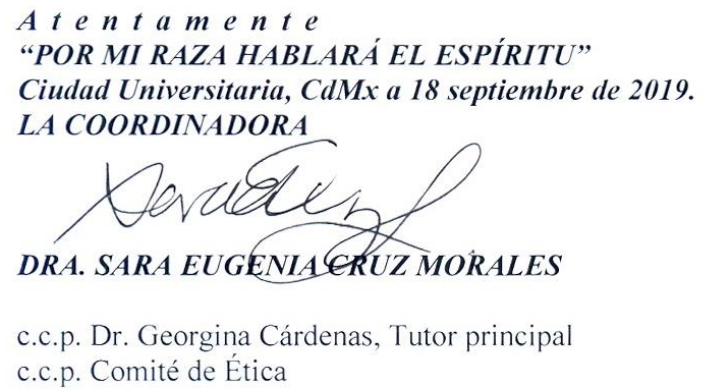

DRA. SARA EUGENIACRUZ MORALES

c.c.p. Dr. Georgina Cárdenas, Tutor principal c.c.p. Comité de Ética 